\title{
Dirección de crecimiento, posición y tamaño de los maxilares en mordidas abiertas esqueléticas de patrón esquelético clase II y III
}

Growth direction, position and size of the jaws in open bite of skeletal class II and III malocclusion

\begin{abstract}
Resumen
El objetivo del estudio fue determinar la dirección de crecimiento, posición y tamaño de la maxila y mandíbula de mordidas abiertas, de patrón esquelético clase II y III. Metodología. Este estudio es observacional, descriptivo y transversal. La población estuvo conformada por 100 radiografías cefalométricas laterales de pacientes con diagnóstico clínico de mordida abierta, el muestreo fue no probabilístico, por conveniencia. Se utilizó el programa Nemoceph para realizar el análisis de Ricketts y conocer los valores de las variables de estudio. La muestra consistió de 36 radiografías de mordidas abiertas esqueléticas. Los datos obtenidos fueron analizados con el test chi cuadrado, a un nivel de confianza del 95\%, equivalente a 1.96 y un margen de error del $5 \%$ y la prueba estadística paramétrica. Resultados. De las 36 mordidas abiertas, el $72.2 \%$ era clase II y el $27.8 \%$ clase III de patrón esquelético, con las siguientes características: los dos grupos en su mayoría mostraron dirección de crecimiento normodivergente en la maxila, y en la mandíbula todos presentaron crecimiento hiperdivergente. Con respecto a la posición, ambos grupos presentaron posición normal de la maxila pero y se diferenciaron en el tamaño encontrándose una maxila de tamańo normal para el grupo de clase II y un tamańo aumentado en el grupo de clase III. Con respecto a la mandíbula, podemos decir que en ambos grupos presentaron tamaño disminuido y se diferenciaron en la posición encontrándose una posición retruída para el grupo de clase II y una posición normal en el grupo de clase III. Conclusion. No hay diferencia significativa en la dirección de crecimiento, posición y tamańo de la maxila y mandíbula en los patrones esqueléticos de clase II y III $(\mathrm{p}<0.05)$.
\end{abstract}

Palabras clave: Mordida abierta, análisis de Ricketts

\begin{abstract}
The aim of the study was to determine the growth direction, position and size of the maxilla and mandible with skeletal open bite Class II and III. Methodology: This is an observational, descriptive and cross-sectional investigation. The population consisted of 100 lateral cephalometric radiographs of patients with clinical diagnosis of skeletal open bite. The sample was not probabilistic for convenience. The Nemoceph program was used for Ricketts analysis to get the values of variables. The sample consisted of 36 radiographs of skeletal open bites. The data obtained were analyzed with the chi-square test at a confidence level of $95 \%$ equivalent to 1.96 and a margin of error of $5 \%$. Results: Of the 36 open bites, $72.2 \%$ were class II and $27.8 \%$ Class III skeletal pattern. The two groups showed mostly normodivergent growth direction in the maxilla but all the mandibles showed hyperdivergent growth. In relation to position, both groups had normal position of the maxilla, but differed in size, being normal size for class II group and increased size for Class III. With respect to mandible, both groups showed decreased size, but differences in position, being a retruded position for class II group and a normal position in class III group. Conclusion: There were no significant difference, $\mathrm{p}<0.05$, in the growth direction, position and size of maxilla and mandible in skeletal patterns of class II and class III.
\end{abstract}

Keywords: Open bite; Ricketts' analysis.

\section{Introducción}

A lo largo del tiempo, las mordidas abiertas han sido asunto predilecto para el ortodoncista. Se han puntualizado dos orígenes de la mordida abierta: dentaria y esquelética; la primera, generalmente relacionada a problemas verticales desarrollados por hábitos de- letéreos como la succión digital, interposición lingual, interposición labial, hábitos posturales y respiración oral; la segunda, mordida abierta esquelética, relacionada a uno o más factores del sistema craneofacial que se han desarrollado anormalmente durante el crecimiento, debido a la complejidad

\section{Artículo Original}

\author{
Lisbeth Pérez Cahuaya ${ }^{1}$, \\ Karla Valdez Castro ${ }^{1}$, \\ Roxana Delgado Llancari', \\ Luis Pérez Vargas², \\ Carlos Tisnado Florián²
}

1. Facultad de Odontología de la Universidad Nacional Mayor de San Marcos, Perú.

2. Unidad de Posgrado, Segunda Especialidad en Ortodoncia de la Facultad de Odontología de la Universidad Nacional Mayor de San Marcos, Perú.

\section{Correspondencia}

C.D. Lisbeth Pérez Cahuaya

Jr. Los Palmitos 488. Urb. Las Violetas,

Lima 36, Perú

Correo electrónico: lipeca25@gmail.com

\section{Coautores}

Valdez: karlamicaro15@hotmail.com

Delgado: burbuja 17_5@hotmail.com

Pérez: lfperezvargas@gmail.com

Tisnado: itisnado@hotmail.com de esta última. El conocimiento de las características y la elaboración de un adecuado diagnóstico son primordiales para lograr un tratamiento estable de mordida abierta que podría implicar desde una simple aparatología fija hasta un procedimiento quirúrgico complejo $^{1,2}$. 
Para decidir el enfoque terapéutico con más probabilidad de éxito, es necesario analizar la forma de crecimiento. Las maloclusiones de mordida abierta se clasifican mediante un adecuado análisis cefalométrico. En la mordida abierta esquelética la altura facial anterior es excesiva, especialmente al nivel del tercio inferior, mientras que la altura posterior (altura de la rama) es corta. La base mandibular suele ser estrecha y a menudo presenta una escotadura antegonial. La sínfisis es estrecha y alargada, y la rama ascendente es corta. El ángulo gonial (en particular la parte inferior) es grande y el patrón de crecimiento es vertical ${ }^{3,4}$.

La dirección de los vectores de crecimiento condiciona la expresión del crecimiento de la cara que, a través de la manifestación de sus condicionantes genéticas y también por las modificaciones inducidas a propósito de las alteraciones funcionales, durante el período de crecimiento del paciente, pueden terminar por representarse clínicamente como mordidas abiertas de diferentes magnitudes y asociadas a rostros con tercio inferior más o menos altos ${ }^{5}$.

Estas variaciones del crecimiento vertical están manifestadas en parámetros de diferentes estudios cefalométricos que nos sirven de referencia en la definición y descripción de las características faciales. En la cefalometría de Ricketts, por ejemplo, encontramos medidas como la altura facial inferior, la inclinación del plano mandibular, el arco mandibular y la inclinación del plano biespinal, que nos ayudan a reconocer, estimar la magnitud del conflicto y el compromiso de las distintas partes del esqueleto afectado ${ }^{8}$.

Por lo tanto, en las mordidas abiertas estructurales o de origen esquelético, siempre habrá un compromiso, más o menos significativo, de aquellas estructuras que se ven condicionadas en su crecimiento por las alteraciones funcionales, como son los rebordes alveolares y la propia dentición, debido a que el compromiso primario afecta la estructura basal ${ }^{9,10}$.

La importancia de dicho trabajo radica en el diagnóstico, ya que el análisis de la forma y dirección de crecimiento es necesario para determinar el enfoque terapéutico con más probabilidad de éxito.

El objetivo de esta investigación es determinar la dirección de crecimiento, posición y tamaño de la maxila y mandíbula de mordidas abiertas de patrón esquelético clase II y III.

\section{Materiales y método}

Este estudio es de tipo descriptivo, retrospectivo y transversal, realizado entre los meses de junio-agosto del ańo 2015. La selección de la población fue de manera no probabilística, por conveniencia y estuvo conformada por radiografías cefalométricas laterales de 100 pacientes con mordida abierta dentaria que acudieron a la clínica de consulta privada de ortodoncia "SMILES", del doctor Fernando Pérez Vargas, las cuales fueron clasificadas en mordida abierta esquelética mediante el análisis de Ricketts, utilizando el programa Nemoceph obteniendo así una muestra de 36 radiografías cefalométricas laterales, que fueron agrupadas en 2 grupos según patrón esquelético: clase II y clase III mediante el ángulo ANB de Steiner y el ángulo de la convexidad de Ricketts. En estos dos grupos se analizaron mediante cinco medidas para describir la mandíbula: eje facial, profundidad facial, ángulo del plano mandibular, altura facial inferior, arco mandibular. Estas, además de definir forma, tamaño, posición mandibular, relación intermaxilar vertical, son útiles para la obtención del biotipo facial del paciente y dos factores para describir el maxilar superior: convexidad facial y profundidad maxilar, que completan el análisis del patrón esqueletal en sentido sagital. La muestra estuvo conformada por 11 varones y 25 mujeres, con rango de edad entre 12 y 29 años.

El ángulo del eje facial nos permitió expresar la dirección de crecimiento de la mandíbula ya que según la literatura no cambia con la edad, su norma clínica es de $90^{\circ}+1-3^{\circ}$. Cuando la medida es menor de $90^{\circ}$ (ángulo más cerrado a la norma), se habla de un "eje facial abierto" y corresponde a un biotipo facial dolicofacial. Al medirse como ya se dijo el ángulo posterior, la expresión "apertura” está referida a una apertura de la mordida y descenso de la mandíbula. Por el contrario, "eje facial cerrado" significa que el ángulo aumenta su valor. A diferencia de la apertura, está describiendo un cierre de la mordida y un ascenso de la mandíbula. Corresponde a un patrón braquifacial. A su vez, el ángulo de la profundidad facial nos permitió evaluar el biotipo facial del paciente; su norma clínica es de $87^{\circ}+/-3^{\circ}$, medidas inferiores a la norma indicaron biotipo dolicofacial y medidas mayores indicaron biotipo braquifacial. El ángulo del plano mandibular nos indica la inclinación del cuerpo mandibular, su norma es de $26^{\circ}+/-4^{\circ}$. Cuando este ángulo es mayor que la norma, estamos en presencia de un paciente dolicofacial con musculatura débil y tendencia a la mordida abierta. Los tipos braquifaciales con musculatura potente y mordida profunda tienen valores menores. El ángulo de la altura facial inferior no cambia con la edad, su norma clínica es $47^{\circ}$ $+/-3^{\circ}$, un ángulo mayor que la norma indica divergencia entre la mandíbula y el maxilar superior y corresponde a un patrón dolicofacial con musculatura débil y con tendencia a mordida abierta esqueletal. A la inversa, valores menores indican patrones braquifaciales, con musculatura fuerte y generalmente con sobremordida profunda. El ángulo del arco mandibular describe la forma de la mandíbula, su valor es de $26^{\circ}+/-4^{\circ}$, un ángulo grande indica una mandíbula fuerte y cuadrada, característica del patrón braquifacial, un ángulo menor representa una mandíbula con rama corta, forma obtusa y un patrón de crecimiento vertical (dolicofacial). Al analizar los resultados de estos cinco ángulos, nos permitió evaluar la dirección de crecimiento, posición y tamańo de la mandíbula.

Por otra parte, la norma clínica del ángulo de la convexidad facial es de $+2 \mathrm{~mm}+/-2$, esta medida describe la relación de ambos maxilares en sentido sagital. Una convexidad mayor que la norma indica un patrón esqueletal de clase II, mientras que una convexidad negativa nos indica un patrón esquelético de clase III. El ángulo de la profundidad del maxilar tiene una norma clínica de $90^{\circ}+/-3^{\circ}$ e indica la localización del maxilar superior en sentido anteroposterior, valores superiores indican una protusión esqueletal del maxilar superior. Un ángulo menor de $90^{\circ}$, por el contrario, nos habla de una retrusión esqueletal del maxilar superior. La evaluación de ambos ángulos nos permitió determinar nuestro objetivo de conocer la dirección de crecimiento, posición y tamańo del maxilar superior.

Asimismo, se evalúo la altura maxilar (Norma clínica $53^{\circ}+/-3^{\circ}$ ) que, al igual que el plano mandibular, es útil para el diagnóstico de las mordidas abiertas esqueléticas. En este caso los valores serán menores que la norma clínica. Los resultados se basaron en la norma clínica de cada ángulo estudiado; valores mayores, normales y menores esta nos indicaron valores aumentados, normales y disminuidos correspondientemente.

El procedimiento fue realizado por un único operador, quien pasó por un proceso de calibración para realizar el aná- 
lisis y la evaluación de las radiografías cefalometrías laterales.

\section{Resultados}

El presente trabajo determinó la dirección de crecimiento, posición y tamaño de los maxilares en 36 mordidas abiertas esqueléticas de patrón esquelético clase II y III, de los cuales el $72.2 \%$ fue de clase II esquelética y el $27.8 \%$ de clase III esquelética (Tabla 1). El análisis estadístico utilizado fue la prueba estadística no paramétrica Chi cuadrado para conocer si las variables estudiadas se encontraban relacionadas y se utilizó el programa estadístico SPSS.

La prueba Chi cuadrado no mostró significancia en el tamaño, posición, dirección de crecimiento de la maxila y mandíbula entre los patrones esqueléticos de clase II y III, $\mathrm{p}>0.05$.

Se observó la maxila de tamaño normal para el grupo de clase II esquelética $(81 \%)$ y un tamańo aumentado en el grupo de clase III esquelética (60\%) (Tabla 2.).

Ambos grupos presentaron en su mayoría una posición normal de la maxila. Clase II esquelética (73\%) y clase III esquelética (60\%) (Tabla 3).

Ambos grupos presentaron en su mayoría una dirección del crecimiento normodivergente en la maxila. Clase II esquelética (69\%) y clase III esquelética (60\%) (Tabla 4).

Ambos grupos presentaron tamaño disminuido de la mandíbula. Clase II esquelética $(69 \%)$ y clase III esquelética (60\%) (Tabla 5).

Se observó una posición retruida de la mandíbula para el grupo de clase II esquelética $(69 \%)$ y una posición normal en el grupo de clase III esquelética (60\%) (Tabla 6).

Se observó un crecimiento hiperdivergente de la mandíbula en ambos grupos. Clase II esquelética (100\%) y clase III esquelética (100\%) (Tabla 7).

Tabla 1. Frecuencia de las mordidas abiertas

\begin{tabular}{ll}
\hline Patrón esquelético & $\mathbf{f} \%$ \\
\hline Clase II & $\mathbf{2 6 7 2 \%}$ \\
Clase III & $1028 \%$ \\
Total & $36100 \%$ \\
\hline
\end{tabular}

Tabla 2. Tamańo de la maxila

\begin{tabular}{lccl}
\hline Patrón & Disminuido & Normal & Aumentado \\
\hline Clase II & $14 \%$ & $2181 \%$ & $415 \%$ \\
Clase III & $220 \%$ & $220 \%$ & $660 \%$ \\
Total & $38 \%$ & $264 \%$ & $1028 \%$ \\
\hline
\end{tabular}

Tabla 3. Posición de la maxila

\begin{tabular}{llcl}
\hline Patrón & Retruida & Normal & Aumentada \\
\hline Clase II & $312 \%$ & $1973 \%$ & $415 \%$ \\
Clase III & $220 \%$ & $660 \%$ & $220 \%$ \\
Total & $514 \%$ & $2569 \%$ & $617 \%$ \\
\hline
\end{tabular}

Tabla 4. Dirección de crecimiento de la maxila

\begin{tabular}{lll}
\hline Patrón & $\begin{array}{l}\text { Normo } \\
\text { Divergente }\end{array}$ & $\begin{array}{l}\text { Hiper } \\
\text { Divergente }\end{array}$ \\
\hline Clase II & $1869 \%$ & $831 \%$ \\
Clase III & $660 \%$ & $440 \%$ \\
Total & $2467 \%$ & $1233 \%$ \\
\hline
\end{tabular}

Tabla 5. Tamańo de la mandíbula

\begin{tabular}{llcl}
\hline Patrón & Disminuido & Normal & Aumentado \\
\hline Clase II & $1869 \%$ & $519 \%$ & $312 \%$ \\
Clase III & $660 \%$ & $00 \%$ & $440 \%$ \\
Total & $267 \%$ & $514 \%$ & $719 \%$ \\
\hline
\end{tabular}

Tabla 6. Posición de la mandíbula

\begin{tabular}{lcll}
\hline Patrón & Retruida & Normal & Aumentada \\
\hline Clase II & $1869 \%$ & $831 \%$ & $00 \%$ \\
Clase III & $00 \%$ & $660 \%$ & $440 \%$ \\
Total & $1850 \%$ & $149 \%$ & $411 \%$ \\
\hline
\end{tabular}

Tabla 7. Dirección de crecimiento de la mandíbula

\begin{tabular}{cc}
\hline Patrón & Hiper divergente \\
\hline Clase II & $\mathbf{2 6 1 0 0 \%}$ \\
Clase III & $\mathbf{1 0 1 0 0 \%}$ \\
Total & $36100 \%$ \\
\hline
\end{tabular}

\section{Discusión}

En 1974, el Dr. Young H. Kim diseñó el indicador de profundidad de sobremordida vertical (Overbite Depth Indicator ODI). Este es la suma aritmética del ángulo del plano A-B al plano mandibular, y el ángulo del plano palatino al plano horizontal de Frankfort. La norma es de $74.5^{\circ}$ con una desviación estándar de 6.07 . Un valor de $68^{\circ}$ o menor indica una mordida abierta esquelética o tendencia a ella ${ }^{11}$.

Zou y col. (2001) investigaron los diferentes tipos craneofaciales de mordida abierta anterior. Los pacientes con mordida abierta anterior fueron clasificados en 5 tipos: dentoalveolar, rotación mandibular $\mathrm{CW}$, cara larga, rotación maxilar CCW y clase III esqueletal ${ }^{12}$.

Stefan y col. encontraron que un incremento en la altura facial inferior durante el tratamiento tiende a disminuir la profundidad de sobremordida. El aumento excesivo en la altura de la sínfisis puede asegurar recidiva ${ }^{13}$.

Haralabakis (1994) evaluó las características cefalométricas que contribuyen al desarrollo de mordida abierta en adultos tridimensionalmente con ra- diografía lateral de cráneo y posteroanterior ${ }^{14}$.

Tanaka (1990) estudió las características morfológicas de mordidas abiertas clase I y II ${ }^{15}$.

En este estudio, la dirección de crecimiento de la mandíbula en el 100\% de los pacientes fue hiperdivergente, lo que explica la tendencia a mordida esquelética abierta y se asemeja a los resultados de estudios publicados por otros autores, tales como Acuña G y Col. ${ }^{7}$ Asimismo, con respecto a la dirección de crecimiento de la maxila, el presente estudio mostró que la clase II (69\%) y clase III esquelética (60\%) presentaron crecimiento normodivergente de la maxila, lo que podría explicarnos en que la mayoría de casos el problema se encuentra a nivel de la mandíbula. Este resultado se asemeja a las características encontradas por Frankel R.6.

No existen muchos antecedentes de investigaciones descriptivas sobre mordida abierta con relación a la dirección y tamańo de los maxilares; sin embargo, es notorio que en las investigaciones mencionadas, al igual que en esta, el intento de clasificar la mordida abierta según distintos parámetros juega un papel primordial en el diagnóstico y la toma de decisión para el tratamiento ortodóntico u ortodóntico-quirúrgico.

En este estudio no se encontró, en general, diferencias entre maxilar y mandíbula en cuanto al crecimiento, tamańo y posición en ambos patrones esqueléticos, por ello se sugiere ampliar la muestra en futuros estudios.

Se sugiere la realización de estudios posteriores enfocados al patrón esquelético en mordida, abierta, ya que este refleja directamente el comportamiento vertical de las bases óseas y, por tanto, la zona de origen de esta maloclusión.

\section{Conclusiones}

Aunque solo un número limitado de muestra fue disponible para el estudio debido a la escasa frecuencia relativa de esta condición, 36 casos de mordida abierta representaron la razonable muestra de una serie de conclusiones que pueden extraerse del presente estudio. Todos los pacientes dentro de los dos grupos, clase II y III, mostraron tendencia de crecimiento hiperdivergente mandibular.

La mordida abierta esquelética fue asociada al patrón esquelético II, con una posición más retruida del mentón. En la mayoría se observaron problemas solo 
en mandíbula mientras que en dos cefalometrías se observaron problemas tanto en la maxila como en la mandíbula; lo que nos dio a conocer que el principal problema etiológico de esta muestra de mordida abierta esquelética se daba en la mandíbula y que su dirección de crecimiento fue hiperdivergente.

\section{Referencias bibliográficas}

1. Alimere, HC. Thomazinho A, Felício C M. Anterior open bite: a formula for the differential diagnosis (original title: Mordida aberta anterior: uma fórmula para o diagnóstico diferencial). Pró-Fono Revista de Atualização Científica, Barueri (SP). 2005;17(3):367-374.

2. Arriola GLE, Flores MC. Molar heights and incisor inclinations in adults with Class II and Class III skeletal open-bite malocclusions. American Journal of Orthodontics and Dentofacial Orthopedics. 2014;145(3):325-332.

3. Oliveira AC, Almeida Pordeush I, Silva Torres C, Torres M. Feeding and nonnutritive sucking habits and prevalence of open bite and crossbite in children/adolescent with Down
Syndrome. Angle Ortthodontist. 2010;80(4): 748-52.

4. Cozza P, Baccetti T, Franchi L, Toffol L, McNamara JJr. Mandibular Changes produced by Functional appliances in Class II Malocclusion: A sistematic Review. AJO. 2008; (5): 8 .

5. Houston WJB. A textbook of orthodontics. Gran Bretaña. Ed. Wright. Second edition. 1998.

6. Cangialosi TJ. Skeletal morphologic features of anterior open bite. Am J Orthod. 2008; 5:28-36.

7. Acuña Dávalos G, Ballesteros Lozano M, Oropeza Sosa G. Descripción cefalométrica del patrón facial en mordida abierta esqueletal. Revista Odontológica Mexicana. 2013; 17 (1):15-19.

8. Marwat HJ, Amin B, Khan A. Frequency of anterior open bite patients reporting to AFID, RAWALPINDI. Pakistan Oral \& Dental Journal. 2009; 281(1): 71-4.

9. Proffit RW. Contemporary treatment of dentofacial deformity. 1era. Ed. St. Louis, Missouri: Mosby, Inc, 2003: 464-491.
10. Clark WJ. Twin block functional therapy. 2nd. Ed. Fife UK: Mosby, Inc. 2002: 165-191.

11. Silva R. Aplicación práctica de los indicadores de profundidad de sobre mordida vertical, displasia anteroposterior e índice de extracción. UNAM, FES Zaragoza 2004.

12. Mitani H. Early application of chincap therapy to skeletal class III malocclusion. Am J Orthop Dentofac Orthop 2002; 121(6) 584- 585 .

13. Beckman S, Segner D. Changes in alveolar morphology during open bite treatment and prediction of treatment result. European Orthodontics Sociaty 2002; 24: 391-406.

14. Ngan P. Biomechanics of maxillary expansion and protaction in class III patients. Am J Orthop Dentofac Orthop 2002; 121(6) 582- 583.

15. Saadia M, Torres E. Vertical changes in class III patients after maxillary protraction with expansion in the primary and mixed dentitions. Pediatr Dent 2001; 23; 125-130. 